\title{
Molecular-Weight Dependence of Intramacromolecular End-to-End Photodimerization Rate of $\alpha, \omega$-Dianthryl- polystyrene in Dilute Solution
}

\author{
Hideharu UshikI, Kimiko HiRAYANAGI, ${ }^{*}$ Yoichi Sindo, ${ }^{*}$ \\ Kazuyuki HORIE, ${ }^{* *}$ and Itaru MITA** \\ Laboratory of Chemistry, Faculty of General Education, \\ Tokyo University of Agriculture and Technology, \\ 3-5-8, Saiwai-cho, Fuchu-shi, Tokyo 183, Japan \\ *Department of Chemistry, Faculty of Science, Toho University, \\ Miyama, Funabashi, Chiba 274, Japan \\ **Institute of Interdisciplinary Research, Faculty of Engineering, \\ The University of Tokyo, 4-6-1, Komaba, Meguro-ku, \\ Tokyo 153, Japan
}

(Received April 27, 1983)

\begin{abstract}
The intramacromolecular photodimerization rate of a pair of terminal groups of $\alpha, \omega$-dianthrylpolystyrene (A-PS-A) was measured for the polymers with various degrees of polymerization, $N$, at high dilution. The extent of intramacromolecular photodimerization of A-PSA during continuous irradiation by a high-pressure mercury lamp was determined from the change in fluorescence intensity of the anthryl groups attached to the polymer chain ends. The apparent rate constant $K_{\mathrm{F}}$ for the polymer end-to-end cyclization of A-PS-A in benzene at $30 \pm 1^{\circ} \mathrm{C}$ was found to be proportional to $N^{-n}$ with $n=1.4 \pm 0.2$ for the range of $N=280-3000$.

KEY WORDS $\alpha, \omega$-Dianthrylpolystyrene / Photodimerization / Diffusion-

Controlled Reaction / Polymer End-to-End Cyclization / Excimer

Formation Rate Constant / Triplet Probe Method / Fluctuation Volume /

Equilibrium Distribution of End-to-End Distance
\end{abstract}

The chemical reactivity of reactive groups in a polymer system is known to be distinguished from that of corresponding model reactions between small molecules. The reaction rate of reactive groups attached to polymer chains is affected by the dynamical motion of polymer chains in a diffusioncontrolled reaction system, ${ }^{1}$ and by the thermodynamical equilibrium behavior of polymer chains in an activation-controlled reaction system. ${ }^{2}$ Hence, in the former case, the apparent rate may be expected to be influenced by the lifetime of the excited reactive groups attached to the polymer chains because an excited reactive group can react only within the excited lifetime.

Photodimerization of various chromophores has been reported by many authors. ${ }^{3-7}$ The photodimerization of anthracene and 9-methylanthracene is known to proceed via a singlet excimer state. Moreover, the assumed diffusion-controlled reac- tion has been proved for the photodimerization of 9-methylanthracene in a fluid solution by Tanaka and Osugi. ${ }^{8}$ A schematic diagram of the photophysical processes of $\alpha, \omega$-dianthrylpolystyrene is shown in Figure 1. The photodimerization of excited anthryl groups attached to polystyrene is completed within a short lifetime $(\tau=4.6-6.1 \mathrm{~ns}),{ }^{9}$ since it proceeds via a singlet excimer state between a pair of terminal groups of $\alpha, \omega$-dianthylpolystyrene. The photodimerization of anthrylpolystyrene differs from the activation- and diffusion-controlled intra- and inter-macromolecular reaction in a fluid solutions, as reported earlier, using triplet probe with a lifetime of the order of micro- or milli-seconds. ${ }^{10-15}$

Our previous study ${ }^{16}$ on the intramacromolecular end-to-end photo-dimerization of $\alpha$-mono- and $\alpha, \omega$ dianthrylpolystyrene (abbreviated PS-A and A-PSA) in a fluid solution by a nitrogen laser pulse 


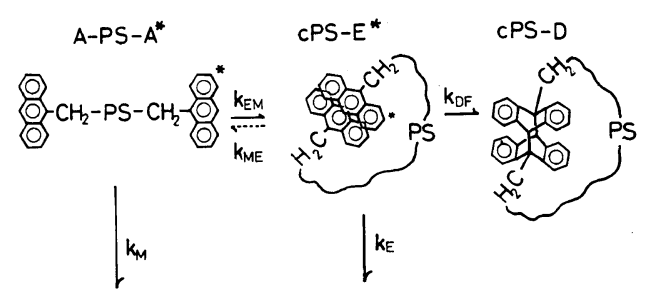

Figure 1. Schematic diagram of a photophysical process for the diffusion-controlled intramacromolecular end-to-end photodimerization of A-PS-A.

showed that the rate constant $k_{\text {intra }}$ for the polymer end-to-end cyclization of A-PS-A in butanone was proportional to $N^{-n}$ with $n=0.3$ for the range of $N=110-2600$, where $N$ is the degree of polymerization. But this experiment with a nitrogen laser pulse involved a few problems get to be solved. One is that the concentration of the polymer ([A-PS$\left.\mathrm{A}]=1.25 \times 10^{-4}-1.0 \times 10^{-5} \mathrm{M}\right)$ in the above study was not sufficiently low to eliminate all the effects of the intermacromolecular reaction, since the rate of intramacromolecular end-to-end photodimerization of A-PS-A for the range of high degree of polymerization $(N=640-2600)$ in the previous study was substantially independent of the degree of polymerization. Another problem is that butanone as the solvent may act as a weak sensitizer for anthryl groups under the irradiation of a nitrogen laser pulse.

In the present paper, the rate of the intramacromolecular end-to-end photodimerization of A-PS-A was measured for the range of $N=280-300^{\circ}$ in benzene, good solvent, at $30 \pm 1^{\circ} \mathrm{C}$ and the apparent rate constants for the diffusion-controlled intramacromolecular reaction for polymers with a high degree of polymerization were obtained as functions of $N$ of polystyrene. The effects of the different lifetime of the probes attached to polymer ends on the molecular-weight dependence of rate constants for diffusion-controlled intramacromolecular endto-end reactions are briefly discussed in the following.

\section{EXPERIMENTAL}

$\alpha, \omega$-Dianthrylpolystyrene (S-PS-A) was prepared in a manner similar way to that reported previously. ${ }^{10,11}$ Styrene was polymerized anionically in tetrahydrofuran (THF) with sodium-naphthalene
Table I. Characteristics of $\alpha, \omega$ dianthrylpolystyrenes

\begin{tabular}{lccrc}
\hline Polymer & $M_{n}$ & $M_{w} / M_{n}$ & $\begin{array}{c}N \text { of } \\
\text { PS chain }\end{array}$ & $\begin{array}{c}\% \text { anthryl } \\
\text { group at a } \\
\text { chain end }\end{array}$ \\
\hline APS280A & $3.0 \times 10^{4}$ & 1.59 & 280 & 100 \\
APS300A & $3.1 \times 10^{4}$ & 1.84 & 295 & 92 \\
APS375A & $3.9 \times 10^{4}$ & 3.24 & 375 & 100 \\
APS640A & $6.7 \times 10^{4}$ & 1.45 & 638 & 100 \\
APS780A & $8.1 \times 10^{4}$ & 1.24 & 774 & 100 \\
APS1300A & $1.4 \times 10^{5}$ & 1.42 & 1292 & 100 \\
APS2600A & $2.8 \times 10^{5}$ & 1.19 & 2665 & 78 \\
APS3000A & $3.1 \times 10^{5}$ & 1.24 & 2963 & 91 \\
\hline
\end{tabular}

as the initiator under a high vacuum, and the resultant living polystyryl dianions were end-capped with 9-chloromethylanthracene prepared in advance from 9-anthraldehyde via 9-hydroxymethylanthracene.

The number-average molecular weight $\left(M_{n}\right)$ of APS-A and molecular-weight distributions $\left(M_{w} / M_{n}\right)$ were measured with a Toyo Soda HLC-802UR gel permeation chromatograph (GPC) at $40^{\circ} \mathrm{C}$ in tetrahydrofuran. The anthryl group content (A) at the chain ends was determined from UV absorption spectra using a Shimadzu MPS-5000 spectrophotometer. Pertinent data are shown in Table I.

Sample solutions in a degassed and sealed rectangular quartz cell were irradiated continuously by a $450 \mathrm{~W}$ high-pressure mercury lamp (Ushio UM-452). Hard glass and/or Pyrex glass plates as filters were set in the apparatus thermostated at $30 \pm 1{ }^{\circ} \mathrm{C}$. The wavelengths at $50 \%$ transmittance of two Pyrex plates, a hard glass plate, and two Pyrex plates along with a hard glass plate were 328,335 , and $346 \mathrm{~nm}$, respectively. The extent of photodimerization of the anthryl groups in the solutions whose concentrations were of the order of $10^{-6} \mathrm{M}$ was determined from the change in intensity of the fluorescence spectra measured with a JASCO FP550 spectrofluorometer. Dotite-Luminasol-grade benzene was used as the solvent in the photodimerization measurements.

\section{RESULTS AND DISCUSSION}

\section{Intramacromolecular End-to-End Photodimerization Analysis of A-PS-A}

Changes in the $I_{\mathrm{F}} / I_{\mathrm{F}}^{0}$ ratio with irradiation time 
brought about by the high-pressure mercury lamp are shown in Figure 2, where $I_{\mathrm{F}}$ is the fluorescence intensity of 9-methylanthracene (MA) at arbitrary irradiation time $t$ in benzene and the superscript 0 designates the initial stage. The reaction rate of the photodimerization of MA in a degassed and sealed cell was slower than that in air of under rough nitrogen bubbling condition, indicating some contribution of oxidation to the consumption of MA. The sample solutions for all subsequent experiments were thus deaerated by several freeze-pump-thaw cycles under high vacuum in the cells which were then sealed off.

To confirm the occurrence of the macromolecular end-to-end photodimerization of the anthryl groups attached to polystyrene chain ends, the GPC for PS3300A before and after continuous irradiation with a $337 \mathrm{~nm}$ pulsed nitrogen laser was measured (Figure 3). The results indicate that the photodimerization of PS-A occurs to a considerable extent in this experimental system as was evidenced by the fact that the number-average molecular weight $\left(M_{n}\right)$ of PS3300A after irradiation was about twice that before irradiation, that is, $M_{n}$ and $M_{w} / M_{n}$ for PS3300A before irradiation were $3.4 \times 10^{5}$ and 1.66 , and after irradiation, $8.8 \times 10^{5}$ and 1.25 .

The intramacromolecular end-to-end photodimerization rate of A-PS-A in a very dilute solution (order of $10^{-6} \mathrm{M}$ ) as a first-order reaction was determined by eq 1 ,

$$
\ln \left(I_{\mathrm{F}}^{0} / I_{\mathrm{F}}\right)=K t
$$

where $I_{\mathrm{F}}$ is the fluorescence intensity of anthryl

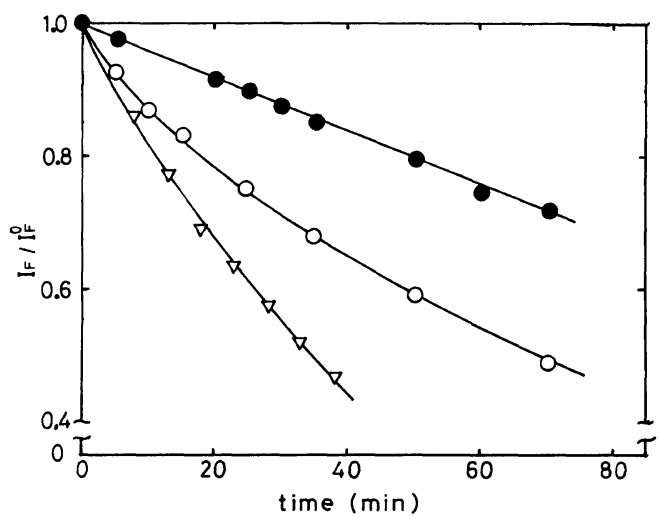

Figure 2. Dependence of $I_{\mathrm{F}} / I_{\mathrm{F}}^{0}$ for the fluorescence intensity of 9-methylanthracene on the irradiation time $t$ using a high-pressure mercury lamp: $(\nabla)$ in air; (O) under the condition of rough nitrogen bubbling condition; (O) in degassed and sealed cell.

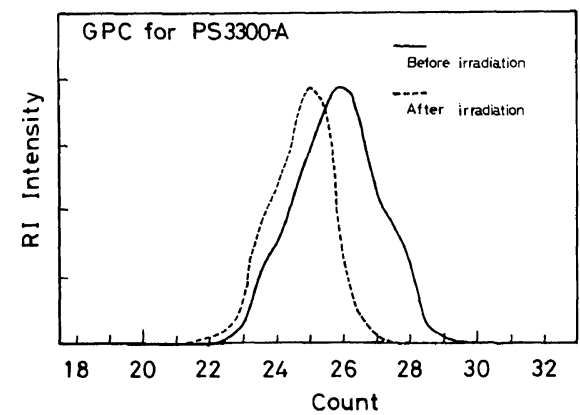

Figure 3. GPC curves for PS3300A before and after continuous irradiation with a $337 \mathrm{~nm}$ pulsed nitrogen laser in butanone at $30^{\circ} \mathrm{C}$ : Solid line, before irradiation; dotted line, after irradiation.

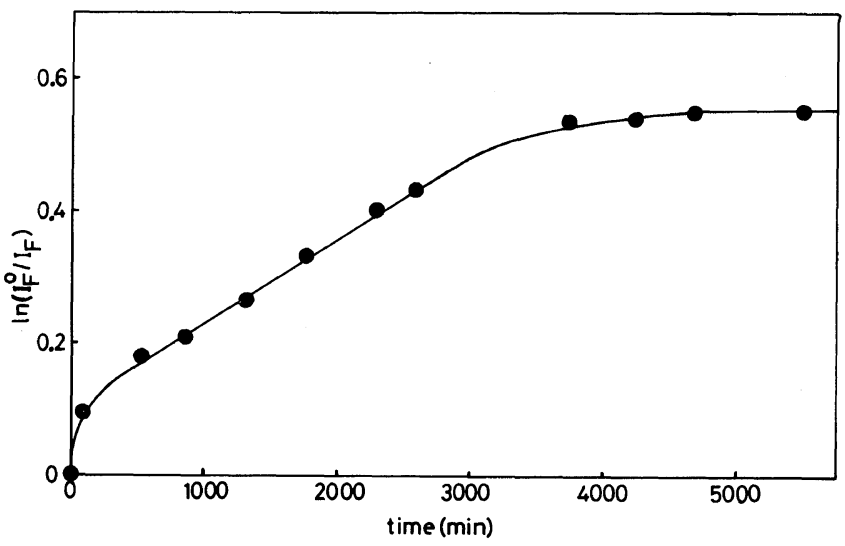

Figure 4. First-order reaction plots using eq 1 for the intramacromolecular end-to-end photodimerization of APS1300A in benzene at $30 \pm 1^{\circ} \mathrm{C}$ with a Pyrex filter. 


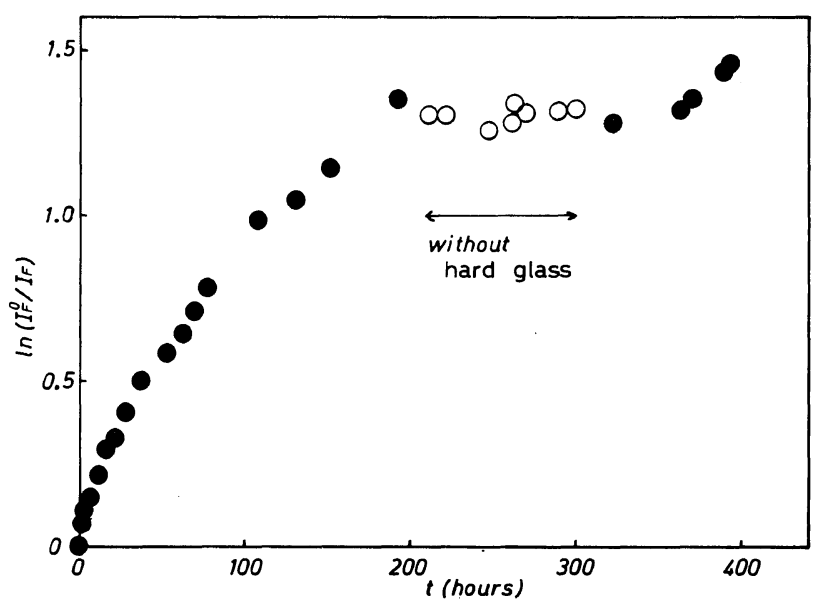

Figure 5. First-order reaction plots for the intramacromolecular end-to-end photodimerization of APS3000A in benzene at $30 \pm 1^{\circ} \mathrm{C}$ using general hard glasses plus Pyrex glass as filters: (O) with general hard glasses plus Pyrex glass as filters; $(\mathrm{O})$ with only Pyrex glass as a filter.

groups after irradiation at arbitrary time $t$, the superscript 0 designates the initial stage, $K$ is the apparent rate constant for the intramacromolecular end-to-end photodimerization of A-PS-A. The plots as a first-order reaction using eq 1 for the intramacromolecular end-to-end photodimerization of APS1300A are shown in Figure 4. The experiment was carried out using only Pylex plates as filters so as to make the irradiation light of wavelength shorter than $320 \mathrm{~nm}$. The relation was not linear, and the curve finally leveled off at a certain value. Consequently, irradiation with only Pyrex glass plates as filters was pressumed to cause the photo-dissociation of dianthryl dimers produced by the photodimerization between the anthryl groups. To confirm the occurrence of a photodissociation reaction in the above experimental system, the photodimerization reaction of A-PSA was carried out using general hard glass and Pyrex glass plates as filters to effectively cut the irradiation light of wavelength shorter than $320 \mathrm{~nm}$ from the high-pressure mercury lamp. The plots as a first-order reaction for the intramacromolecular end-to-end photodimerization of APS1300A in this case are shown in Figure 5. This was not a straight line. While the intensity of incident light through general hard glass and Pyrex glass plates decreased more than that when using only Pyrex glass plates, the apparent intramacromolecular end-to-end photodimeri-

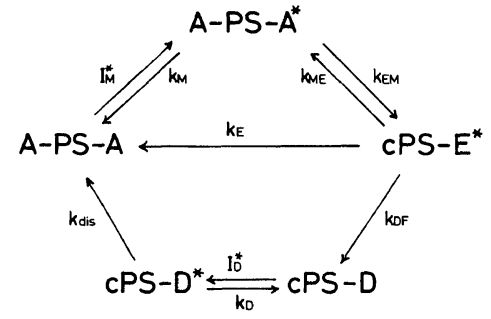

Figure 6. Schematic diagram of photophysical processes for A-PS-A, including a photodissociation process of a dianthryl dimer: A-PS-A, ground state of monomer; A-PS-A*, singlet excited state of monomer; cPS-E*, intramacromolecular end-to-end singlet excimer state; cPS-D, ground state of dimer; cPS-D*, singlet excited state of dimer.

zation rate of A-PS-A under the former condition was faster than that under the latter condition. When the general hard glass plates were removed during the photodimerization of A-PS-A using general hard glass and Pyrex glass plate filters, the rate became quite slow, as evident from Figure 5 , by the open circles. This supports the presumption that irradiation light through the Pyrex glass plates as filters causes to some extent the photodissociation reaction of dianthryl dimers produced by the photodimerization of the anthryl groups attached to the polymer chain end. The schematic diagram of the photophysical and photochemical processes for A-PS-A, including a photo- 
dissociation process of the anthryl dimers, is shown in Figure 6.

\section{Kinetics of Intramacromolecular End-to-End Photo- dimerization of $A-P S$ - $A$}

All subsequent experiments were carried out using Pyrex glass plates as filters, and so the data obtained for the intramacromolecular end-to-end photodimerization of A-PS-A show the influence of the photodissociation reaction of dianthryl dimers following photodimerization of the anthryl groups. The rate for the above reaction system is expressed by eq $2-6$.

$$
\mathrm{d}[\mathrm{A}-\mathrm{PS}-\mathrm{A}] / \mathrm{d} t=-I_{\mathrm{M}}^{*}+k_{\mathrm{M}}\left[\mathrm{A}-\mathrm{PS}-\mathrm{A}^{*}\right]+k_{\mathrm{E}}\left[\mathrm{CPS}-\mathrm{E}^{*}\right]
$$

$$
\begin{aligned}
\mathrm{d}\left[\mathrm{A}-\mathrm{PS}-\mathrm{A}^{*}\right] / \mathrm{d} t= & I_{\mathrm{M}}^{*}-\left(k_{\mathrm{M}}+k_{\mathrm{EM}}\right)\left[\mathrm{A}-\mathrm{PS}-\mathrm{A}^{*}\right] \\
& +k_{\mathrm{ME}}\left[\mathrm{CPS}-\mathrm{E}^{*}\right]
\end{aligned}
$$

$$
\mathrm{d}\left[\mathrm{cPS}-\mathrm{E}^{*}\right] / \mathrm{d} t=k_{\mathrm{EM}}\left[\mathrm{A}-\mathrm{PS}-\mathrm{A}^{*}\right]
$$

$$
-\left(k_{\mathrm{E}}+k_{\mathrm{DF}}+k_{\mathrm{ME}}\right)\left[\mathrm{cPS}-\mathrm{E}^{*}\right]
$$

$$
\mathrm{d}[\mathrm{cPS}-\mathrm{D}] / \mathrm{d} t=-I_{\mathrm{D}}^{*}+k_{\mathrm{DF}}\left[\mathrm{cPS}-\mathrm{E}^{*}\right]+k_{\mathrm{D}}\left[\mathrm{cPS}-\mathrm{D}^{*}\right]
$$$$
+k_{\mathrm{dis}}\left[\mathrm{cPS}-\mathrm{D}^{*}\right]
$$

$$
\mathrm{d}\left[\mathrm{cPS}-\mathrm{D}^{*}\right] \mathrm{d} t=I_{\mathrm{D}}^{*}-\left(k_{\mathrm{dis}}+k_{\mathrm{D}}\right)\left[\mathrm{cPS}-\mathrm{D}^{*}\right]
$$

[A-PS-A], [A-PS-A*], [cPS-E*], [cPS-D], and [cPS$\mathrm{D}^{*}$ ] are the concentrations of the ground state of the monomer, the singlet excited state of the monomer, the intramacromolecular end-to-end singlet excimer, the ground state of the dimer, and the singlet excited state of the dimer, respectively, $k_{\mathrm{EM}}$ is the rate constant for the intramacromolecular end-toend excimer formation, $k_{\mathrm{M}}$, the sum of rate constants for fluorescence, nonradiative transition, and intersystem crossing of the singlet monomer, $k_{\mathrm{E}}$, the sum of rate constants for excimer emission and nonradiative transition of the excimer, and $k_{\mathrm{ME}}, k_{\mathrm{DF}}$, and $k_{\mathrm{dis}}$, the rate constants for dissociation of the excimer, dimerization, and photodissociation of the excited dimer, respectively. $k_{\mathrm{D}}$ is the sum of rate constants for fluorescence, nonradiative transition, and intersystem crossing of the excited dimer, and $I^{*}$ is the rate for formation of excited molecules with $\mathrm{M}$ and $\mathrm{D}$ as a suffix denoting the monomer and dimer.

When $\varepsilon_{\mathrm{M}}[\mathrm{A}-\mathrm{PS}-\mathrm{A}]$ and $\varepsilon_{\mathrm{D}}[\mathrm{cPS}-\mathrm{D}]$ are less than about 0.1 , the rate for the formation of excited molecules by the incident light in the light path within $1 \mathrm{~cm}$ can be adequately approximated by eq 7 and 8 ,

$$
\begin{aligned}
& I_{\mathrm{M}}^{*}=I_{\mathrm{M}}^{0}\left\{1-\exp \left(-2 \varepsilon_{\mathrm{M}}[\mathrm{A}-\mathrm{PS}-\mathrm{A}]\right)\right\} \simeq 2 I_{\mathrm{M}}^{0} \varepsilon_{\mathrm{M}}[\mathrm{A}-\mathrm{PS}-\mathrm{A}] \\
& I_{\mathrm{D}}^{*}=I_{\mathrm{D}}^{0}\left\{1-\exp \left(-\varepsilon_{\mathrm{D}}[\mathrm{cPS}-\mathrm{D}]\right)\right\} \simeq I_{\mathrm{D}}^{0} \varepsilon_{\mathrm{D}}[\mathrm{cPS}-\mathrm{D}]
\end{aligned}
$$

where $I$ is the intensity of incident light per unit time and $\varepsilon$, the molar extinction coefficient multiplied by 2.3 for anthryl groups and dianthryl dimers (for example, $\varepsilon_{M}=5.3 \times 10^{3}$ at $389 \mathrm{~nm}$ in benzene).

Now we introduce the relation $[\mathrm{A}-\mathrm{PS}-\mathrm{A}]_{t}=$ $[\mathrm{A}-\mathrm{PS}-\mathrm{A}]_{t}^{0}-[\mathrm{cPS}-\mathrm{D}]_{t}$, where $[\mathrm{A}-\mathrm{PS}-\mathrm{A}]_{t}=[\mathrm{A}-$ PSPA $]+[$ A-PS-A*] $+[$ cPS-E* $\quad(\simeq[$ A-PS-A $])$ and $[\mathrm{cPS}-\mathrm{D}]_{t}=[\mathrm{cPS}-\mathrm{D}]+\left[\mathrm{cPS}-\mathrm{D}^{*}\right] \quad(\simeq[\mathrm{cPS}-\mathrm{D}]) . \quad$ The superscript 0 designates the initial concentration of A-PS-A, and the suffix $t$, the concentration of A-PS$A$ and cPS-D not at the time of irradiation with a high-pressure mercury lamp but when measurement of fluorescent intensity by a spectrofluorometer is made. By using a steady state approximation for excited-state species, the decrease rate in the fluorescent intensity of anthryl groups can be obtained from eq $2-8$.

$$
\begin{gathered}
-\mathrm{d}[\mathrm{A}-\mathrm{PS}-\mathrm{A}]_{t} / \mathrm{d} t=\left(K_{\mathrm{F}}+K_{\mathrm{B}}\right)[\mathrm{A}-\mathrm{PS}-\mathrm{A}]_{t} \\
-K_{\mathrm{B}}[\mathrm{A}-\mathrm{PS}-\mathrm{A}]_{t}^{0} \\
K_{\mathrm{F}}=\frac{2 k_{\mathrm{EM}} k_{\mathrm{DF}} \varepsilon_{\mathrm{M}} I_{\mathrm{M}}^{0}}{k_{\mathrm{M}}\left(k_{\mathrm{E}}+k_{\mathrm{DF}}+k_{\mathrm{ME}}\right)+k_{\mathrm{EM}}\left(k_{\mathrm{E}}+k_{\mathrm{DF}}\right)} \\
K_{\mathrm{B}}=k_{\mathrm{dis}} \varepsilon_{\mathrm{D}} I_{\mathrm{D}}^{0} /\left(k_{\mathrm{dis}}+k_{\mathrm{D}}\right)
\end{gathered}
$$

where $K$ is the apparent rate constant with $\mathrm{F}$ and $\mathrm{B}$ the suffixes denoting the forward (photodimerization) and backward (photodissociation) reactions, respectively, for the intramacromolecular end-to-end photoreaction of A-PS-A. To obtain the change in [A-PS-A $]_{t}$ with irradiation time $t$, eq 9 can be integrated to give eq 12 .

$$
\begin{gathered}
\ln \left\{\frac{K_{\mathrm{F}} / K_{\mathrm{B}}[\mathrm{A}-\mathrm{PS}-\mathrm{A}]_{t}^{0}}{\left(1+K_{\mathrm{F}} / K_{\mathrm{B}}\right)[\mathrm{A}-\mathrm{PS}-\mathrm{A}]_{t}-[\mathrm{A}-\mathrm{PS}-\mathrm{A}]_{t}^{0}}\right\} \\
=K_{\mathrm{B}}\left(1+K_{\mathrm{F}} / K_{\mathrm{B}}\right) t
\end{gathered}
$$

For the equilibrium state of the photodimerization and photodissociation reactions, the equation relating the apparent rate constant for the forward reaction with that of the backward is,

$$
[\mathrm{A}-\mathrm{PS}-\mathrm{A}]_{t}^{0} /[\mathrm{A}-\mathrm{PS}-\mathrm{A}]_{t}^{e}=1+K_{\mathrm{F}} / K_{\mathrm{B}}
$$




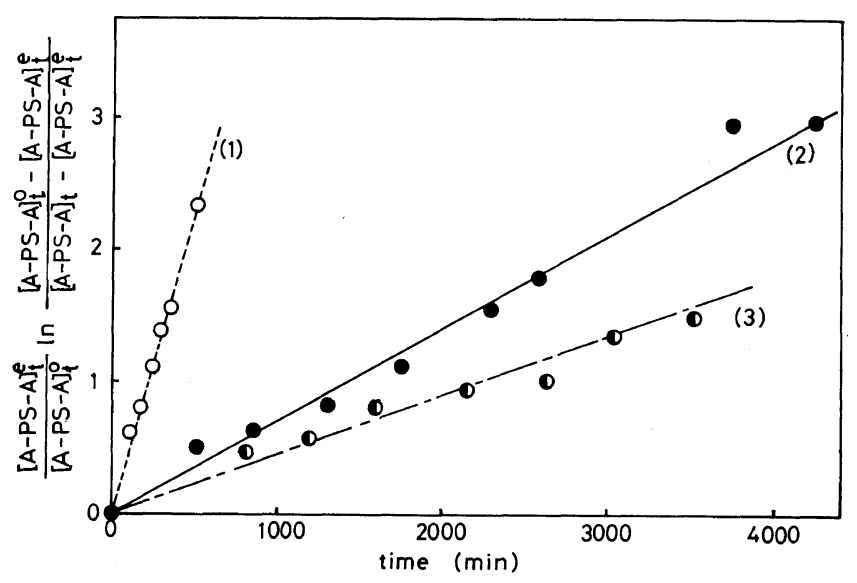

Figure 7. Replots using eq 14 for the intramacromolecular end-to-end photodimerization of APS1300A in benzene at $30 \pm 1{ }^{\circ} \mathrm{C}$ with a Pyrex filter: (1), APS280A $\left(2.5 \times 10^{-6} \mathrm{M}\right) ;(2)$, APS1300A $\left(2.5 \times 10^{-6} \mathrm{M}\right)$; (3), APS3000A $\left(2.5 \times 10^{-6} \mathrm{M}\right)$.

where the superscript $e$ designates the equilibrium point of the forward and backward reactions for the intramacromolecular end-to-end photodimerization of A-PS-A. Consequently, eq 12 is transformed into eq 14 by eq 13 .

$$
\frac{[\mathrm{A}-\mathrm{PS}-\mathrm{A}]_{t}^{e}}{[\mathrm{~A}-\mathrm{PS}-\mathrm{A}]_{t}^{0}} \ln \left\{\frac{[\mathrm{A}-\mathrm{PS}-\mathrm{A}]_{t}^{0}-[\mathrm{A}-\mathrm{PS}-\mathrm{A}]_{t}^{e}}{[\mathrm{~A}-\mathrm{PS}-\mathrm{A}]_{t}-[\mathrm{A}-\mathrm{PS}-\mathrm{A}]_{t}^{e}}\right\}=K_{\mathrm{B}} t
$$

Replots using eq 14 for the intramacromolecular end-to-end photodimerization of APS1300A are shown in Figure 7. The relation was linear as expected. Hence, $K_{\mathrm{F}}$ defined by eq 10 can be easily calculated from eq 13 with the value of $K_{\mathrm{B}}$ obtained from the slope of the straight line in Figure 7. The fluorescent intensity $\left([\mathrm{A}-\mathrm{PS}-\mathrm{A}]_{t}^{e}\right.$ ) at the equilibrium point of the forward and backward reactions for the intramacromolecular end-to-end photodimerization of APS1300A was determined from the constant values of fluorescent intensity within the range from about 4000-6000 minutes.

We can assume $k_{\mathrm{M}} \gg k_{\mathrm{EM}}$, the $K_{\mathrm{F}}$ can be adequately approximated as follows, ${ }^{16}$

$$
K_{\mathrm{F}} \simeq \frac{2 k_{\mathrm{EM}} k_{\mathrm{DF}} \varepsilon_{\mathrm{M}} I_{\mathrm{M}}^{0}}{k_{\mathrm{M}}\left(k_{\mathrm{E}}+k_{\mathrm{DF}}+k_{\mathrm{ME}}\right)}=2 k_{\mathrm{EM}} \tau_{\mathrm{s}} \Phi_{\mathrm{D}} \varepsilon_{\mathrm{M}} I_{\mathrm{M}}^{0}
$$

where $\tau_{\mathrm{s}}$ is the singlet excited lifetime of anthryl groups (4.9-5.5 ns for anthracene and 4.6-6.1 ns for 9-methylanthracene) ${ }^{9}$ and $\Phi_{\mathrm{D}}$, the dimer yield of the anthryl groups from a singlet excimer state.
According to eq 15, the apparent forward rate constant, $K_{\mathrm{F}}$, for the intramacromolecular end-to-end photodimerization of A-PS-A contains many constants $\left(k_{\mathrm{EM}}, \tau_{\mathrm{s}}, \Phi_{\mathrm{D}}, \varepsilon_{\mathrm{M}}\right.$, and $\left.I_{\mathrm{M}}^{0}\right)$, among which, only the rate constant $k_{\mathrm{EM}}$ for the intramacromolecular end-to-end excimer formation can be predicted to be influenced by the degree of polymerization, $N$, of A-PS-A. If the approximations for eq 15 are valid, the molecular-weight dependence of the apparent forward rate constant $K_{\mathrm{F}}$ corresponds to that of the rate constant $k_{\mathrm{EM}}$ for the intramacromolecular endto-end excimer formation of A-PS-A.

\section{Data for Molecular-Weight Dependence of $K_{\mathrm{F}}$ for $A$ -} $P S-A$

The apparent rate constants $K_{\mathrm{F}}$ for the intramacromolecular end-to-end photodimerization of A-PS-A in benzene at $30 \pm 1^{\circ} \mathrm{C}$ are shown in Table II, along with the $K_{\mathrm{B}}$ values and the polymer concentrations studied. Since the apparent rate for the intramacromolecular end-to-end photodimerization of APS3000A at the polymer concentration of $0.078 \%$ agrees well with that at the polymer concentration of $0.039 \%$, the influence of intermacromolecular end-to-end photodimerization of A-PS-A can possibly be eliminated at concentrations less than $2.5 \times 10^{-6} \mathrm{M}$ in the range of $N=280-3000$. All subsequent experiments were carried out at very dilute solution concentrations where only intramacromolecular end-to-end photodimerization takes place. 
Table II. Rate constants for intramacromolecular end-to-end photodimerization of $\alpha, \omega$-dianthrylpolystyrene in benzene at $30 \pm 1^{\circ} \mathrm{C}$

\begin{tabular}{|c|c|c|c|c|c|}
\hline \multirow{2}{*}{ Polymer } & \multicolumn{2}{|c|}{ Concentration } & \multirow{2}{*}{$\frac{K_{\mathrm{F}}}{\mathrm{s}^{-1}}$} & \multirow{2}{*}{$K_{\mathrm{F}} / K_{\mathrm{F}}^{\mathrm{ASP} 280 \mathrm{~A} \mathrm{a}}$} & \multirow{2}{*}{$\frac{K_{\mathrm{B}}}{\mathrm{s}^{-1}}$} \\
\hline & M & $\mathrm{gdl}^{-1}$ & & & \\
\hline APS280A & $2.5 \times 10^{-6}$ & $7.4 \times 10^{-3}$ & $3.4 \times 10^{-5}$ & 1.0 & $3.5 \times 10^{-5}$ \\
\hline APS $300 \mathrm{~A}$ & $2.5 \times 10^{-6}$ & $7.9 \times 10^{-3}$ & $4.0 \times 10^{-5}$ & 1.2 & $3.0 \times 10^{-5}$ \\
\hline APS375A & $2.5 \times 10^{-6}$ & $9.8 \times 10^{-3}$ & $2.6 \times 10^{-5}$ & 0.76 & $3.5 \times 10^{-5}$ \\
\hline APS640A & $2.5 \times 10^{-6}$ & $1.7 \times 10^{-2}$ & $1.6 \times 10^{-5}$ & 0.46 & $1.9 \times 10^{-5}$ \\
\hline APS780A & $2.5 \times 10^{-6}$ & $2.0 \times 10^{-2}$ & $5.4 \times 10^{-6}$ & 0.16 & $7.5 \times 10^{-6}$ \\
\hline APS $1300 \mathrm{~A}$ & $2.5 \times 10^{-6}$ & $3.4 \times 10^{-2}$ & $4.7 \times 10^{-6}$ & 0.14 & $3.7 \times 10^{-6}$ \\
\hline APS2600A & $2.5 \times 10^{-6}$ & $6.8 \times 10^{-2}$ & $1.9 \times 10^{-6}$ & 0.056 & $4.3 \times 10^{-6}$ \\
\hline APS $3000 \mathrm{~A}$ & $2.5 \times 10^{-6}$ & $7.8 \times 10^{-2}$ & $1.4 \times 10^{-6}$ & 0.041 & $6.7 \times 10^{-6}$ \\
\hline APS $3000 \mathrm{~A}$ & $1.3 \times 10^{-6}$ & $3.9 \times 10^{-2}$ & $1.8 \times 10^{-6}$ & 0.053 & $6.9 \times 10^{-6}$ \\
\hline
\end{tabular}

${ }^{\text {a }} K_{\mathrm{F}}^{\mathrm{APS} 280 \mathrm{~A}}$, the value of rate constant for the intramacromolecular end-to-end photodimerization of APS280A.

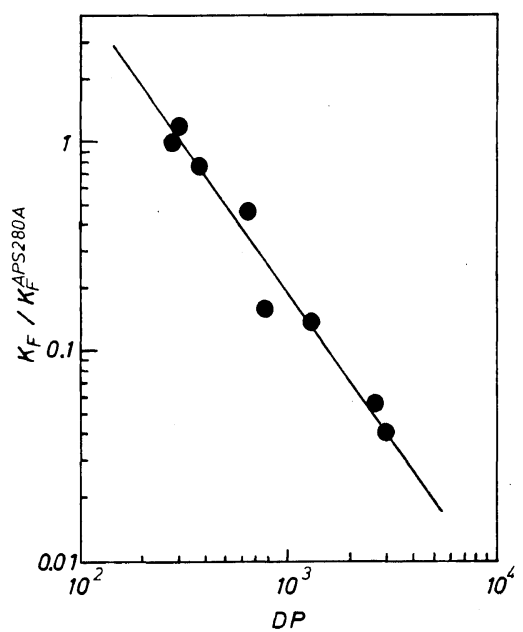

Figure 8. Molecular-weight dependence of $K_{\mathrm{F}} /$ $K_{\mathrm{F}}^{\mathrm{APS} 280 \mathrm{~A}}$ for the intramacromolecular end-to-end photodimerization of A-PS-A in benzene at $30 \pm 1{ }^{\circ} \mathrm{C}$.

The ratios $K_{\mathrm{F}} / K_{\mathrm{F}}^{\mathrm{APS} 280 \mathrm{~A}}$ of the apparent forward rate constants obtained by the intramacromolecular end-to-end photodimerization for A-PS-A in benzene at $30 \pm 1^{\circ} \mathrm{C}$ are plotted in Figure 8 as a function of the degree of polymerization, $N$. Figure 8 shows that the ratios $K_{\mathrm{F}} / K_{\mathrm{F}}^{\mathrm{APS280A}}$ range from 1.0 to 0.0041 and decrease with increasing $N$. The dependence of $K_{\mathrm{F}} / K_{\mathrm{F}}^{\text {APS280A }}$ on $N$ in benzene (good solvent) follows the relationship $K_{\mathrm{F}} / K_{\mathrm{F}}^{\mathrm{APS} 280 \mathrm{~A}}=$ $Z N^{-n}$, where $n=1.4 \pm 0.2$, and $Z$ is a constant. Since the apparent forward rate constant $K_{\mathrm{F}}$ is proportional to the rate constnat $k_{\mathrm{EM}}$ for the intramacromolecular end-to-end excimer formation of A-PS-A according to eq 15 , the ratio $K_{\mathrm{F}} / K_{\mathrm{F}}^{\mathrm{APS} 280 \mathrm{~A}}$ is expressed by eq 16 .

$$
\begin{array}{r}
K_{\mathrm{F}} / K_{\mathrm{F}}^{\mathrm{APS} 280 \mathrm{~A}}=k_{\mathrm{EM}} / k_{\mathrm{EM}}^{\mathrm{ASP280A}}=Z N^{-n} \\
(n=1.4 \pm 0.2)
\end{array}
$$

In Table II, the ratios $K_{\mathrm{B}} / K_{\mathrm{B}}^{\mathrm{APS} 280 \mathrm{~A}}$ of the apparent backward rate constants obtained for the intramacromolecular photo-dissociation of cyclic polymer range from 1.0 to 0.19 and decrease with increasing $N$. This indicates that light having a wavelength shorter than about $300 \mathrm{~nm}$ may be absorbed by polystyrene (intramacromolecular filter effect), there is the possibility of intramacromolecular entangling behavior or cage effect from the photodissociation reaction of the cyclic polymer.

\section{Molecular-Weight Dependence of $K_{\mathrm{F}}$ for $A-P S-A$}

In this research attention has been directed to the molecular-weight dependence of intramacromolecular end-to-end reactions of polymers in dilute solution. Based on the results obtained so far, an attempt was made to determine the rate constant of interaction of terminal chromophores and to relate it to the behavior of $k_{\mathrm{EM}}$, the rate constant of the dynamically controlled end-to-end cyclization for a polymer of monodisperse molecular weight.

The rate constants for the diffusion-controlled end-to-end cyclization of polystyrene in a goodsolvent are proportional to $N^{-n}$ with $n=1.4 \pm 0.2$ in benzene at $30 \pm 1{ }^{\circ} \mathrm{C}$ for the range of $N=280-3000$ (excited singlet lifetime: $4.6-6.1 \mathrm{~ns}$ ), $n=1.6 \pm 0.1 \mathrm{in}$ toluene for the range $N=28-960$ (excited singlet 
lifetime: about $200 \mathrm{~ns}),{ }^{17}$ and $n=1.0 \pm 0.06$ in benzene for the range $N=110-3000$ (excited triplet lifetime: about $0.2-1.0 \mathrm{~ms}),{ }^{13}$ The rate constants for the end-to-end excimer formation of bis((1pyrenylmethoxy)carbonyl)alkanes reported by Nishijima et al. ${ }^{18}$ are proportional to $N^{-n}$ with $n=$ 1.5 in methyltetrahydrofuran at $-60^{\circ} \mathrm{C}$ for the range $N=2-13$ (excited singlet lifetime: about $250 \mathrm{~ns})$. Triplet-state probes attached to polymer chain ends were quite useful for measuring the rate of diffusion-controlled end-to-end cyclization for a very long polymer chain. ${ }^{12,13}$ But in spite of the short lifetime of the excited singlet state, photodimerization of anthryl groups attached to polymer chain ends has a special merit. The reaction rates of the photodimerization of A-PS-A can be specified in terms of the accumulation of a dynamically controlled end-to-end excimer formation process between a pair of terminal groups of the polymer and thus their measurement can be carried out at a sufficiently low polymer concentration $(2.5 \times$ $10^{-6} \mathrm{M}$ ) and high degree of polymerization.

A comparison of data obtained by the triplet probe method ${ }^{12,13}$ with the results of this work leads to the conclusion that the chain length dependence of the rate constant for a diffusion-controlled intramacromolecular end-to-end reaction may be influenced by the excited lifetime of the chromophores attached to polymer chain ends. The chain length dependence of the rate constant, $k_{\text {intra }}$, for a diffusion-controlled irreversible intramacromolecular end-to-end reaction, as determined by the long-excited-lifetime probe (triplet probe) can be explained by assuming the occurrence of rapid fluctuations in chain end segments. ${ }^{13}$ According to our preceding paper, ${ }^{13}$ the diffusion-controlled endto-end reaction takes place when the end-to-end distance, $r$, becomes smaller than the fluctuation diameter, $r_{\mathrm{f}}$, of chain ends since the motion of the chain ends is very rapid and free within the sphere of fluctuation. Consequently, the rate constant, $k_{\text {intra }}$, for a diffusion-controlled irreversible reaction is obtained as,

$$
\begin{aligned}
& k^{\text {intra }}=Z N^{-1.0} \quad\left(\text { for } V_{\mathrm{r}}=V_{\mathrm{f}}>V_{\mathrm{et}}\right) \\
& k_{\text {intra }}=Z N^{-1.5} \quad \text { (for } V_{\mathrm{r}} \pm V_{\mathrm{et}} \text { ) }
\end{aligned}
$$

where $V_{\mathrm{r}}$ and $V_{\mathrm{f}}$ are the reaction and fluctuation volumes of a chromophore attached to a polymer chain end, and $V_{\text {et }}$ is the volume for the energy transfer between a pair of terminal groups of polymers. In the present work, the excited lifetime of a terminal group as a probe was very short compared to that of a triplet probe. Since the excited singlet anthryl groups attached to polystyrene chain ends can react with other anthryl groups only within a very short-excited-lifetime (about $4.6-6.1 \mathrm{~ns}$ ), ${ }^{9}$ a chain end group can move only in vicinity of the initial end-to-end distribution of the polymer prior to the reaction. In the present experimental system, the effects of fluctuation of a chromophore attached to a polymer chain end can hardly be detected in the measurements of the chain length dependence of $k_{\text {intra }}$ due to the very short lifetime of the excited state. Thus, it may be expected that $k_{\text {intra }}$ obtained by the very short-excited-lifetime probe method depends on $N$ as $k_{\text {intra }}=Z N^{-1.5}$ (see eq 18). This expection roughly agrees with our present experimental results obtained by the photodimerization of A-PS-A (see Figure 8).

Under our experimental conditions, the kinetics for the intramacromolecular end-to-end photodimerization of A-PS-A is quite complicated by the inclusion of anthryl dimer photodissociation reactions. If the intramacromolecular photodimerization rates of a pair of terminal groups, not photodissociated after photodimerization can be measured in our experimental system, these kinetics can be analyzed easily and the data will be more reliable. In order to further clarify the molecular-weight dependence of rate constants of intramacromolecular end-to-end photodimerization, such data will be of value.

In conclusion, it may be stated that the molecular-weight dependence of rate constants of the intramacromolecular end-to-end cyclization of linear polystyrene obtained by photodimerization method is proportional to $N^{-n}$ with $n=1.4 \pm 0.2$ in benzene at $30 \pm 1^{\circ} \mathrm{C}$ for the range $N=280-3000$. The dependence of $k_{\text {intra }}$ obtained by the very shortexcited-lifetime probe method on $N$ reflects the equilibrium distribution of the end-to-end distance of polymers. Hence, the chain length dependence of $k_{\text {intra }}$ in a semi-concentrated polymer solution should warrant additional study.

\section{REFERENCES}

1. I. Mita, Ann. New York Acad. Sci., 366, 62 (1981).

2. M. A. Winnik, Chem. Rev., 81, 491 (1981). 
3. H. Bouas-Laurent, A. Castallen, and J. P. Desvergne, Pure Appl. Chem., 52, 2633 (1980).

4. P. P. Wells and H. Morison, J. Am. Chem. Soc., 97, 157 (1975).

5. P. J. Wagner and D. J. Bucheck, J. Am. Chem. Soc., 92, 181 (1970).

6. I. M. Hartmann, W. Hartmann, and G. O. Schenck, Chem. Ber., 100, 3146 (1967).

7. P. Wilairat and B. Selinger, Aust. J. Chem., 21, 733 (1968).

8. F. Tanaka and J. Osugi, Rev. Phys. Chem. Jpn., 42, 85 (1972).

9. S. L. Murov, "Handbook of Photochemistry," Marcel Dekker Inc., New York, 1973.

10. H. Ushiki, K. Horie, A. Okamoto, and I. Mita, Polym. J., 11, 691 (1979).
11. H. Ushiki, K. Horie, A. Okamoto, and I. Mita, Polym. J., 12, 35 (1980).

12. H. Ushiki, K. Horie, A. Okamoto, and I. Mita, Polym. J., 13, 191 (1981).

13. K. Horie, W. Schnabel, I. Mita, and H. Ushiki, Macromolecules, 14, 1422 (1981).

14. K. Horie and I. Mita, Macromolecules, 11, 1175 (1978).

15. I. Mita, K. Horie, and M. Takeda, Macromolecules, 14, 1428 (1981).

16. H. Ushiki, K. Horie, A. Okamoto, and I. Mita, Polym. Photochem., 1, 303 (1981).

17. A. E. C. Redpath and M. A. Winnik, Ann. New York Acad. Sci., 366, 75 (1981).

18. T. Kanaya, K. Goshiki, M. Yamamoto, and Y. Nishijima, J. Am. Chem. Soc., 104, 3580 (1982). 